\title{
New experimental apparatus for the study of the Bénard-Rayleigh problem
}

\author{
J. Moreno, J. Jimenez, A. Córdoba, E. Rojas, and M. Zamora \\ Departamento de Termologia. Facultad de Fisica, Universidad de Sevilla, Sevilla, Spain \\ (Received 30 July 1979; accepted for publication 1 October 1979)
}

\begin{abstract}
A new experimental system to measure the equivalent thermal conductivity of a liquid with regard to the Bénard-Rayleigh problem was constructed. The liquid is enclosed within walls of polymethylmethacrylate between two copper plates in which there are thermocouples to measure the difference in temperature between the lower and upper surfaces of the layer of liquid. Heat flux is measured by means of a linear heat fluxmeter consisting of 204 thermocouples in series. The fluxmeter was calibrated and the linear relationship that exists between the heat flux and the emf generated was verified. The thermal conductivity of the polymethylmethacrylate employed was measured and measurements of the equivalent conductivity in cylindrical boundaries of two silicone oils were made. The critical value of the temperature difference and the contribution of the convective process to the transmission of heat were determined.
\end{abstract}

\section{INTRODUCTION}

In the well-known Bénard-Rayleigh problem ${ }^{1}$ a horizontal layer of fluid confined by two conducting plates and heated uniformly from below the formation of dissipative structures takes place when the critical value of the Rayleigh number is surpassed. The Rayleigh number is defined by the equation

$$
R=g \alpha \Delta T d^{3} / \nu \kappa
$$

where $g$ is the acceleration of gravity, $\Delta T$ the difference in temperature between the plates, $d$ the thickness of the fluid layer, $\alpha$ the coefficient of thermal expansion of the fluid, $\nu$ its kinematic viscosity, and $\kappa$ its thermal diffusivity.

The above phenomenon is accompanied by a variation of the Nusselt number

$$
N u=Q / Q
$$

where $Q$ is the total heat flux through the fluid layer and $Q_{r}$ the heat flux which would occur if there were only conduction. For values greater than the first critical Rayleigh number the structures develop, and new structural forms may occur.

A direct method of studying the Bénard-Rayleigh problem consists of finding the relationship between $\mathrm{Nu}$ and $R$, which experimentally necessitates the simultaneous measurements of the heat flux through the fluid layer and of the difference in temperature applied to it. A number of experimental apparatus and results have been reported in the literature. ${ }^{2-8}$ Nevertheless, there are discrepancies on some questions and on others there is not sufficient information.' For that reason, it seems desirable to develop new experimental apparatus which would permit more precise measurements to be made of the variables relevant to this problem and, in particular, of the heat flux. The present work is an attempt to improve the measurement of this important parameter.

\section{EXPERIMENTAL APPARATUS}

The apparatus consists essentially of two well-differentiated parts: the lower one $L$ contains the heating apparatus and the upper part $\mathrm{H}$ contains the heat fluxmeter and cooling system. The liquid is situated between the two parts, surrounded laterally by a wall of polymethylmethacrylate (PMM) (Fig. 1).

The part $\mathrm{L}$ is formed of a copper plate $\mathrm{A} 85 \times 120 \times 10$ $\mathrm{mm}$, the upper face of which is polished flat and smooth, and in whose lower face is embedded a heating element $(145 \Omega$ ) of manganin wire $0.1 \mathrm{~mm}$ in diameter. An opening of $1 \mathrm{~mm}$ in diameter exists in the underside of the plate in the geometric center into which a chromelconstantan thermocouple is inserted, the junction of which is lodged a few tenths of a millimeter from the upper surface, which is not perforated.

The plate $\mathrm{A}$ is supported by eight steel and celotex rods which maintain it at a distance of $5 \mathrm{~mm}$ from the adiabatic screen $P$. This screen is an uncovered copper box $115 \times 150 \times 24 \mathrm{~mm}$ with walls $10 \mathrm{~mm}$ in thickness. Its interior horizontal surface has embedded in it an electric heating element $(285 \Omega)$ similar to that of piece A. Three chromel-constantan thermocouples (p) in series are mounted between $A$ and $P$. The adiabatic screen is supported in just the same way in the interior of a steel shell that rests on three screws which are used to level the assembly. The purpose of the adiabatic screen is to avoid heat loss in plate A downwards producing sufficient heat by means of its heating element to ensure at all times that the thermocouples p send no signal.

Part $\mathrm{H}$ consists of a copper plate $B$ of the same dimensions as $\mathbf{A}$ and with an identical arrangement of the central thermocouple $b$, but without a heating element. In this case the polished surface faces downwards.

In plate $A$ three thermocouples of chromel-alumel are placed in a position similar to a. These thermocouples are mounted in series with the reference junc- 
FIG. 1. Schematic diagram of the experimental apparatus.

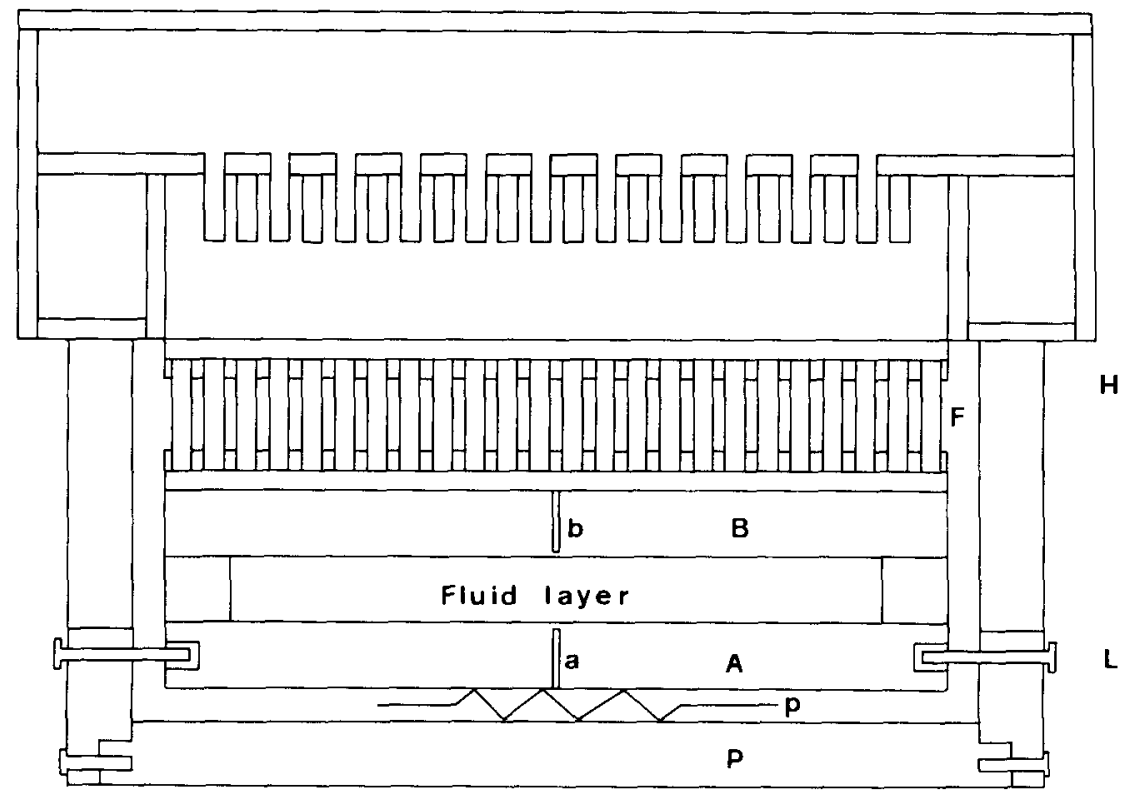

tions in $B$ arranged similarly to $b$. The measurement of difference in temperature between $A$ and $B$ is made by means of these thermocouples in series, using $a$ and $b$ to determine the absolute value of their temperatures.

The heat fluxmeter $F$ is placed on plate $B$. It consists of 204 chromel-constantan thermocouples in series. Each thermocouple consists of cables $3 \mathrm{~mm}$ in diameter and is soldered with silver to copper rectangles $4.5 \times 9.5 \times 3 \mathrm{~mm}$. This assembly of rectangles is cemented to copper plates $85 \times 120 \times 3 \mathrm{~mm}$. The uniform distribution of the sensor elements guarantees uniformity of heat flux throughout the entire system.

A copper plate $85 \times 120 \times 25 \mathrm{~mm}$ is located on the

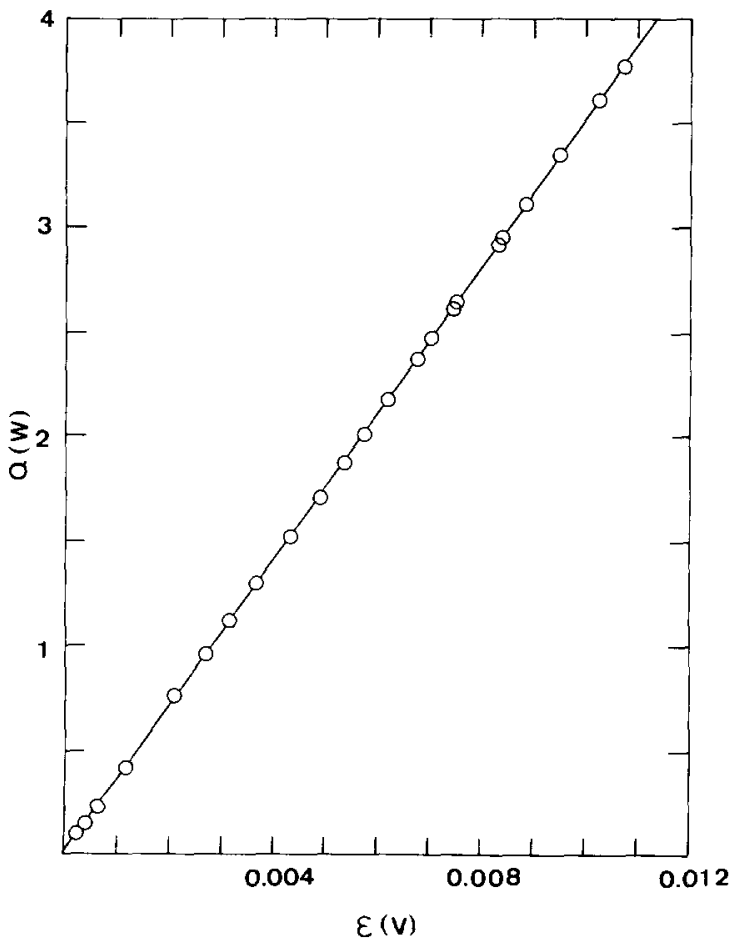

FIG. 2. Calibration of the heat fluxmeter in the range $0-4 \mathrm{~W}$. heat fluxmeter. The upper face of this plate contains channels through which cooling water flows in opposite directions in neighboring channels in order to avoid horizontal gradients of temperature. The upper part is surrounded by a plastic structure which channels the cooling water.

A film of insulating material (Mylar) extends from the edge of the lower plate $A$ to the top of the adiabatic screen, semihermetically sealing the area between them. The part of the apparatus between the edge of the steel box and the plastic structure is also surrounded by insulating material.

Heating occurs by means of several rheostats and two ATAIO 301A power supplies. The cooling water is maintained at a constant temperature by a thermostatic bath which assures a fluctuation of temperature less than $0.05^{\circ} \mathrm{C}$ in the six hours which is the maximum an experiment may last. The measurements of the electrical input to the heating element of plate $\mathrm{A}$, the emf of the differential thermocouples of the adiabatic screen, the thermocouples of plates $A$ and $B$, and the signal of the heat fluxmeter, are made with a modified H.P. 3050B on line data acquisition system, which consists of a $9825 \mathrm{~A}$ calculator, a 3455A digital voltmeter, a 59308A timing generator, a $3495 \mathrm{~A}$ scanner, and a $9862 \mathrm{~A}$ plotter. The heating of the adiabatic screen is regulated by means of an automatic system governed by the calculator according to the signal continuously being received from the thermocouples $\mathrm{p}$.

\section{CALIBRATION}

The theory on which the operation of the fluxmeter is based predicts a linear relationship between the emf it produces $\epsilon$ and heat flux $Q$ when in a stationary state.

The determination of this relationship requires a process of calibration of the heat fluxmeter that is carried out by placing plate $A$ on plate $B$ and simultaneously determining the emf produced by the fluxmeter and the 


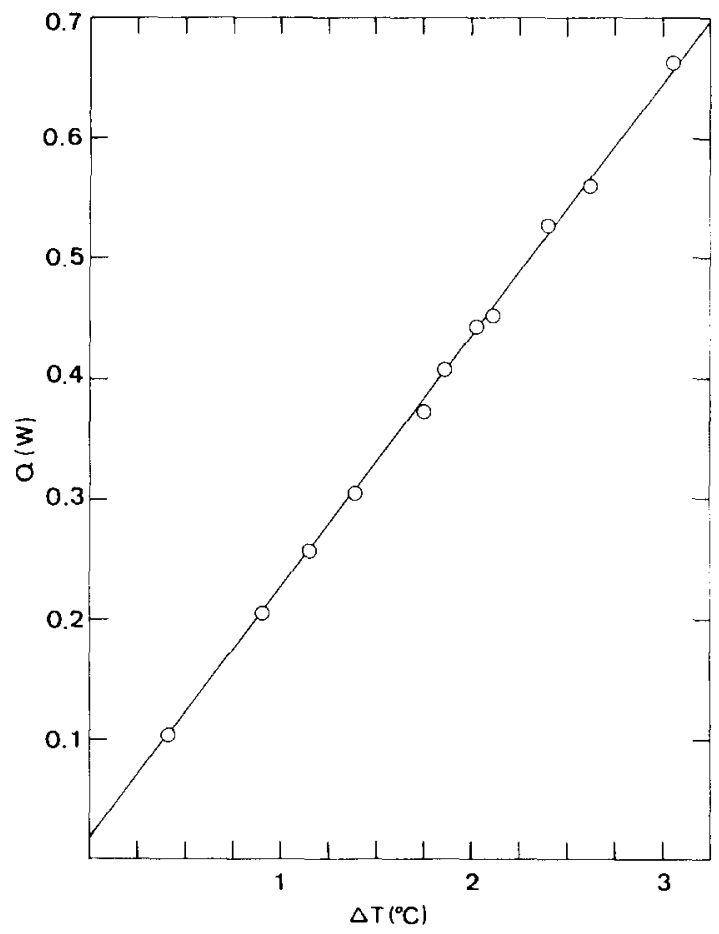

FIG. 3. Measurement of the thermal conductivity of PMM.

output dissipated in A, provided that the differential thermocouples of the adiabatic screen indicate the same temperature in $\mathrm{A}$ as in $\mathrm{P}$. The calibration was carried out in the range of power $0-4 \mathrm{~W}$, the result of which is presented in Fig. 2, where the linearity of the response may be observed.

\section{MEASUREMENT OF THE THERMAL CONDUCTIVITY OF PMM}

Although the experimental apparatus was designed and constructed specifically in order to study the BénardRayleigh problem, it may also be used to measure thermal conductivities.

In order to study the Bénard-Rayleigh problem, the fluid layer which is the object of study is situated between plates A and B laterally surrounded by walls of PMM. Part of the heat which flows between these plates does so through the aforementioned walls by heat conduction. Therefore, it is very important to determine the thermal conductivity of the PMM with the greatest possible precision. To obtain this data we used the same experimental apparatus described above.

A plate of PMM $9.87 \mathrm{~mm}$ thick was placed between A and $B$ and $\Delta T$ and $\epsilon$ were measured when the system had stabilized. The attainment of stability is very slow, especially for high values of $\epsilon$ and $\Delta T$ (up to about six hours) and small fluctuations, due primarily to disturbances produced by the cooling water, always existed. Taking the average value of the measurements, performed each $10 \mathrm{~s}$, over a span of $2 \mathrm{~h}$, a standard deviation of less than $4 \times 10^{-3} \mathrm{~W}$ was obtained. The results are presented in Fig. 3. The value obtained for thermal conductivity is

$$
K=(2.05 \pm 0.04) 10^{-3} \mathrm{~W} /{ }^{\circ} \mathrm{C} \mathrm{cm} .
$$

TABLE 1. Properties of the silicone oils used

\begin{tabular}{lcc}
\hline & Silicone oil I & Silicone oil II \\
\hline Density $\left(\mathrm{g} / \mathrm{cm}^{3}\right)$ & 0.963 & 0.966 \\
Kinematic viscosity $\left(\mathrm{cm}^{2} / \mathrm{s}\right)$ & 0.92 & 2.95 \\
Specific heat $(\mathrm{cal} / \mathrm{g} \mathrm{K})$ & 0.379 & 0.388 \\
Thermal expansion coefficient $\left(\mathrm{K}^{-1}\right)$ & $9.29 \times 10^{4}$ & $9.06 \times 10^{-4}$ \\
Thermal conductivity $(\mathrm{W} / \mathrm{K} \mathrm{cm})$ & $1.60 \times 10^{-3}$ & $1.64 \times 10^{-: 3}$ \\
\hline
\end{tabular}

\section{THE BENARD-RAYLEIGH PROBLEM IN CYLINDRICAL BOUNDARIES}

Subsequently, we proceeded to the study of the onset of convection in a fluid layer heated uniformly from below. Two silicone oils were used, which we will denote I and II, the physical properties of which we measured and present in Table $\mathrm{I}$. We observed a slight variation in these values with temperature, especially as to viscosity. The values shown in Table I are those of the estimated average temperature of the layer of fluid at the threshold of convection.

The fluid was situated in a cylindrical receptacle $65.44 \mathrm{~mm}$ in diameter and $9.87 \mathrm{~mm}$ high, hollowed out of a parallelepiped of PMM, which was placed between the two plates $\mathrm{A}$ and $\mathrm{B}$.

In Fig. 4 the heat flux through the fluid layer is plotted versus the difference in temperature between the plates. The critical values of $\Delta T$ at the onset of convection are:

Silicone oil I: $\quad 2.02 \pm 0.01^{\circ} \mathrm{C}$.

Silicone oil II: $\quad 6.40 \pm 0.01^{\circ} \mathrm{C}$.

The values of thermal conductivity were calculated from the straight line showing $Q$ vs $\Delta T$ resulting from our experimental data before the onset of convection. These are given in Table I.

The critical Rayleigh numbers, $R_{r}$, obtained were

Silicone oil I: $R_{r}=1830 \pm 100$,

Silicone oil II: $R_{r}=1770 \pm 90$

The expected values predicted by Charlson and Sani"

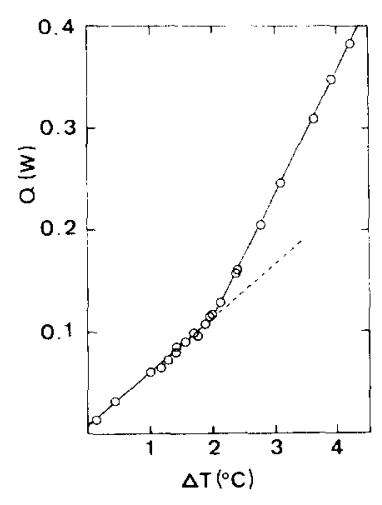

a)

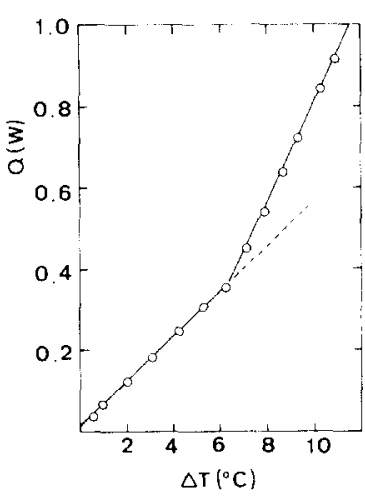

b)
Fig. 4. Heat flux plotted vs temperature difference: (a) silicone oil I; (b) silicone oil II. 


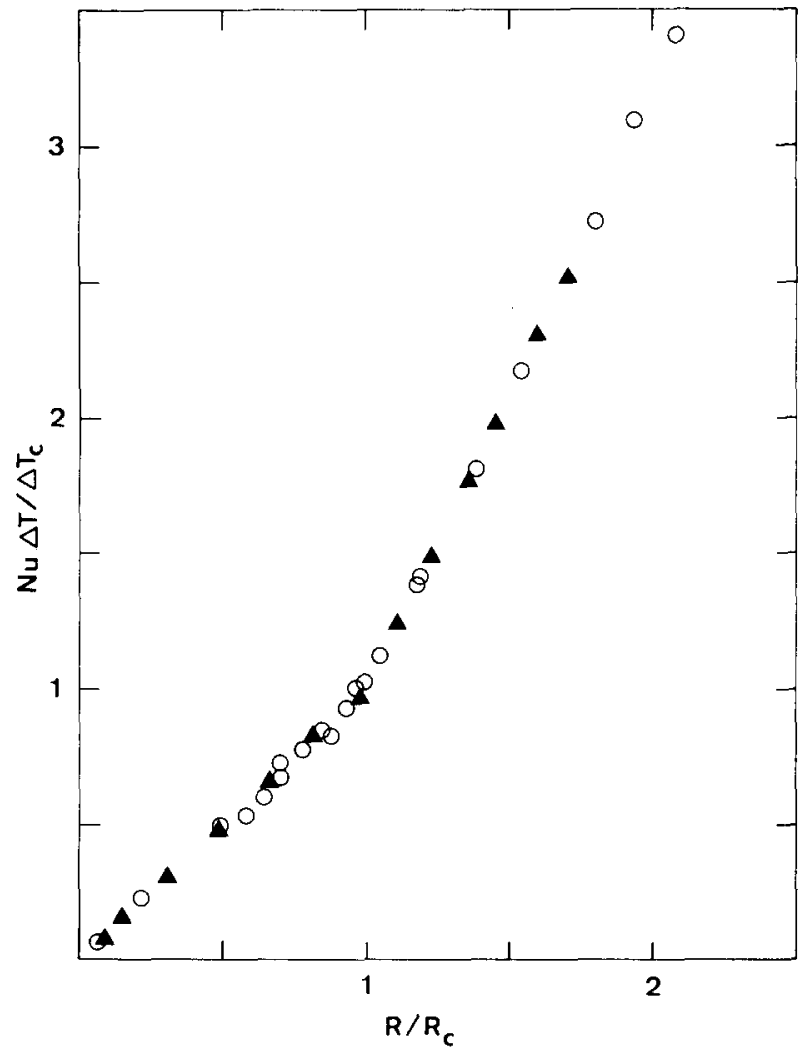

FIG. 5. Adimensional heat flux $N u \Delta T / \Delta T_{r}$ plotted vs normalized Rayleigh number $R / R_{c}:(O)$ silicone oil I; ( $\Delta$ ) silicone oil II.

for a layer of fluid in cylindrical boundaries with an aspect ratio (radius of cylinder/height of cylinder) of 3.25 , as is the case with our apparatus, having adiabatic side walls, is 1753 . For side walls with perfect conductivity it is 1768 .

On the other hand, if $N u \Delta T / \Delta T_{c}$ is plotted versus $R / R_{r}$ (Fig. 5), the results for both silicone oils overlap.

\section{DISCUSSION}

The original contribution of our experimental device is the introduction of a heat fluxmeter which permits a direct measurement of the real heat flux through the fluid layer. The measurements made with this fluxmeter are differential ones and therefore the temperature oscillation of the cooling water affects the measurements. Two precautions were taken to minimize this influence: the first consists of designing junctions of large size, which greatly reduces thermal oscillations; and the second consists of obtaining the average value of the heat flux measurement at sufficiently long intervals of time.

The error in $R_{r}$ derives principally from our imperfect knowledge of the properties of the silicone oils used. In any event, it should be emphasized that the transition from conduction to convection occurs abruptly without observing, within the limits of our apparatus, curvatures in the line $Q$ vs $\Delta T$ as $R_{r}$ is approached.

\section{ACKNOWLEDGMENTS}

The authors wish to express their thanks to M. G. Velarde, P. Bergé and M. Dubois for their comments and suggestions. This work was financially supported by the Comisión Asesora de Investigación Científica y Técnica of the Spanish government, and the French embassy in Spain.

1 E. L. Koschmieder, Adv. Chem. Phys. 26, 177 (1973).

${ }^{2}$ R. J. Schmidt and S. W. Milverton, Proc. R. Soc. London Ser. A 152, 586 (1935).

${ }^{3}$ P. L. Silveston, Forsch. Ing. Wes. 24, 29 and 59 (1958)

${ }^{4}$ H. T. Rossby, J. Fluid Mech. 36, 309 (1969).

${ }^{5}$ R. Krishnamurti, J. Fluid Mech. 42, 295 (1970).

${ }^{6}$ D. R. Caldwell, Rev. Sci. Instrum. 41, 1856 (1970).

${ }^{7}$ E. L. Koschmieder and S. G. Pallas, Int. J. Heat Mass Transfer 17, 991 (1974).

J. R. Booker, J. Fluid Mech. 76, 741 (1976)

" G. S. Charlson and R. L. Sani, Int. J. Heat Mass Transfer 13, $1479(1970)$. 